\title{
Group A Streptococcus: allelic variation, population genetics, and host-pathogen interactions
}

\author{
Sean D. Reid, N ancy P. H oe, Laura M. Smoot, and James M. M usser \\ Laboratory of Human Bacterial Pathogenesis, Rocky M ountain Laboratories, N ational Institute of Allergy and Infectious Diseases, \\ N ational Institutes of Health, 903 South Fourth Street, Hamilton, M ontana, USA \\ Address correspondence to: James M . M usser, Laboratory of H uman Bacterial Pathogenesis, Rocky M ountain Laboratories, National \\ Institute of Allergy and Infectious Diseases, N ational Institutes of Health, 903 South Fourth Street, Hamilton, M ontana 59840, USA. \\ Phone: (406) 363-9315; Fax: (406) 363-9427; E-mail: jmusser@niaid.nih.gov.
}

Group A Streptococcus (GAS) is a Gram-positive bacterium that is responsible for a wide range of human infections, including pharyngitis and tonsillitis, skin infections (impetigo, erysipelas, and cellulitis), sepsis, osteomyelitis, a toxic-shock syndrome, and necrotizing fasciitis (1). The organism is al so responsible for nonsuppurative sequelae such as acute rheumatic fever and acute glomerulonephritis. Globally, GAS causes extensive human morbidity and mortality (1). In the US, approximately 10,000 cases of severe invasive GAS disease occur each year. Direct costs associated with pharyngitis in the US have been estimated to be $\$ 1$ billion annually. Although antibiotic treatment has largely el iminated rheumatic fever and subsequent rheumatic heart disease in N orth America and Western Europe, these crippling forms of heart disease are still very common in the developing world and in certain indigenous populations in developed countries.

The resurgence of GAS as a cause of serious human infections in the US, Europe, and elsewhere in the 1980s and into the 1990s has been thoroughly documented and has heightened public awareness about this organism (1). Disease resurgence, coupled with thelack of a licensed GAS vaccine and ongoing concern about acquisition of penicillin resistance, has stimulated renewed interest in the molecular pathogenesis of GAS disease. The return of GAS as a cause of serious infections was a strong reminder that biomedical science knows relatively little about the evolutionary and other forces that drive temporal variation in bacterial diseasefrequency and severity. GAS's revival has al so taught the importance of understanding the molecular basis of pathogen variation, not merely as a means to discriminate between clinically relevant strains, but to provide data relevant to pathogenesis, host adaptation, and the origin of new pathogenic forms. In this Perspective, we highlight certain aspects of the genetic variation present in GAS, and westress the biomedical relevance of this diversity.
Extensive allelic variation in GAS structural genes Until 1989, most information pertaining to variation in GAS was based on serologic differences in M protein, an antiphagocytic surface moleculethat is a critical virulence factor in many GAS (2). Although approximately $80 \mathrm{M}$ types were recognized, and knowledge of variation in this protein was quite useful for certain kinds of epidemiological studies, serologic classification alone failed to reveal thespecial characteristics of GAS that were associated with certain infection types, such as streptococcal toxic shock syndrome (STSS). The convention of classifying isolates on the basis of one surface antigen was not satisfactory for the study of genomic relationships becauseserologic methods do not ref lect the complexity of the genetic structure of
GAS's revival has also taught the importance of

understanding the molecular basis of pathogen variation, not merely as a means to discriminate between clinically relevant strains, but to provide data relevant to pathogenesis, host adaptation, and the origin of new pathogenic forms. 


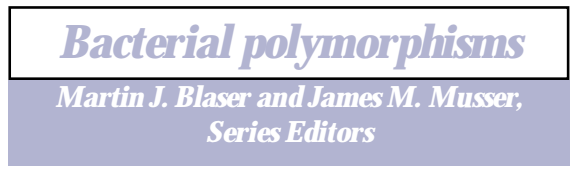

horse for indexing levels of allelic variation in structural genes and estimating levels of chromosomal variation among eukaryotic organisms and bacterial strains $(4,5)$. Thestudy found that allelic variation was extensive in GAS, and, importantly, that more than one-half of thestrains were members of clonally related lineages designated as multilocus electrophoretic type-1 and -2 (ET 1 and ET 2), marked by serotypes M 1 and $M 3$, respectively. M oreover, the analysis identified a strong statistical relationship between the presence of the gene (speA) encoding the superantigen strepto-

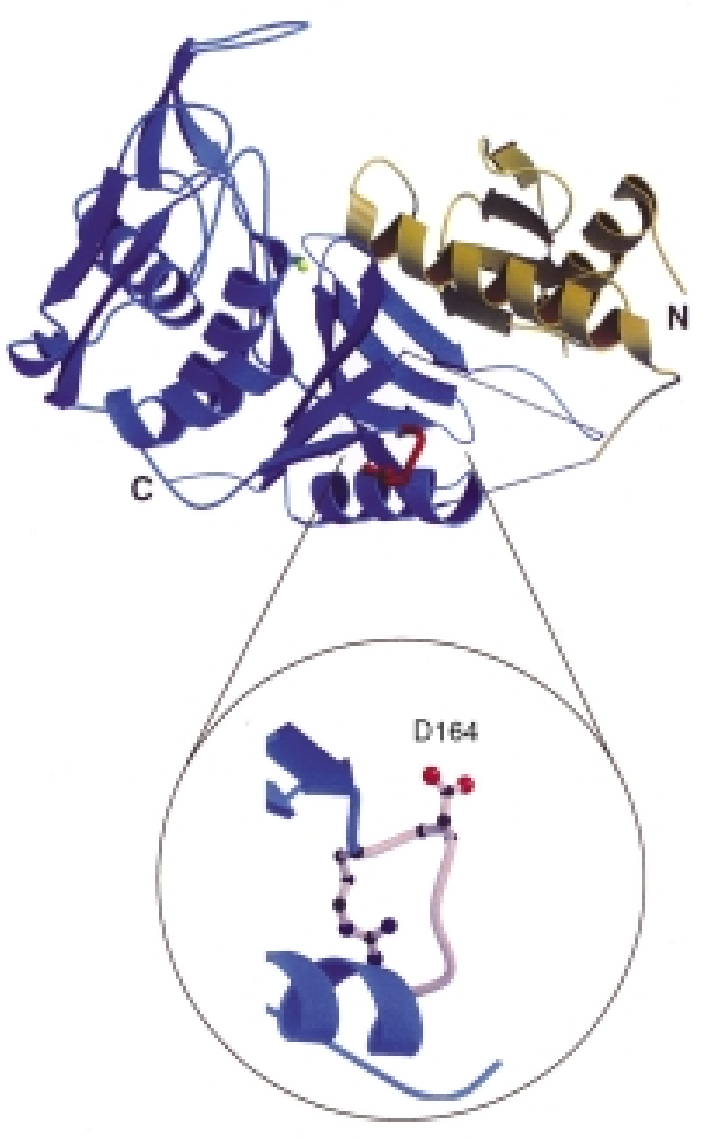

Figure 1

Ribbon diagram of the three-dimensional structure of SpeB, an extracellular cysteine protease virulence factor. The protease domain is shown in blue, the prosegment in brown, the catalytic site cysteine in green, and the RGD loop embellishment in red. Dotted lines indicate flexible regions. The RGD loop is magnified to reveal the exposed Asp-164 residue required for binding of $\alpha_{v} \beta_{3}$ and $\alpha \|_{l b} \beta_{3}$ human integrins. Serotype $M 1$ strains that are abundant causes of human disease contain the RGD motif in the integrin-binding loop, whereas other strains possess an RSD sequence that does not bind integrins (8). N, amino-terminus; C, carboxy-terminus. M odified from ref. 23 with permission. coccal pyrogenic exotoxin A (scarlet fever toxin) and STSS. Hence, analysis of allelic variation in GAS recovered from clinically relevant human infections provided new insight into host-pathogen relationships occurring in nature. Moreover, the findings stimulated investigations resulting in the discovery of three new SpeA variants (see below).

Knowledge of allelic variation in GAS genes, especially those encoding putative or proven virulence factors, expanded rapidly when automated DNA sequencing methods were formulated (Table 1). One common theme revealed by large-scale analysis of virulence gene variation is that several mechanisms have contributed to generating allelic diversity in natural populations of GAS, including point mutations, slipped-strand mispairing, and assortive recombination (6-19).

Theresults of one recent study illustrate the extensive allelic diversity that can be present in GAS. Lukomski et al. (18) identified an extracellular protein with collagen-like characteristics that is present in all GAS. Identification of an extracellular collagenlike protein made by GAS is potentially of considerable interest, given the association of the pathogen with various sequel ae presumed to have an autoimmune component. Theprotein has a cell-wall anchor motif at the carboxy-terminus, variable numbers of contiguous collagen-like Gly-X-X triplet amino acid residue repeats, and a variable amino-terminus. This streptococcal collagen-like $(\mathrm{Scl})$ protein participates in the adherence of GAS to human cells and the pathogenesis of soft tissue infection in a mouse model of human disease. The scl gene was sequenced in 50 strains representing 21 distinct $M$ protein serotypes. The number of contiguous $\mathrm{Gl}$ - $\mathrm{X}$-X motifs ranged from 12 in serotype $M 6$ isolates to 62 in a serotype M 42 organism. The amino acid sequence also varied in the $G \mid y-X-X$ regions, with 50 distinct Gly-X-X sequences identified. Interestingly, $M I$ and $M 18$ organisms had the identical allele which indicates very recent horizontal gene transfer.

The observation that $\mathrm{Scl}$ is an extracellular protein that is potentially subjected to selective pressure in the host suggested that molecular evolutionary genetic analysis would provide insight into the processes shaping variation at thescl locus. The level of selective constraint, a measure of the putative functional importance of a genetic region, was determined for different domains of this locus. The analysis showed that the locus is responding to selective pressure in such a way as to increase the genetic distance between $\mathrm{scl}$ alleles while maintaining the $\mathrm{Gl} y-X-X$ repeat motif. The discovery of a collagen-like gene that encodes a highly variable extracellular protein provides a framework for analysis of the role of structural variation in autoimmune sequelae. 


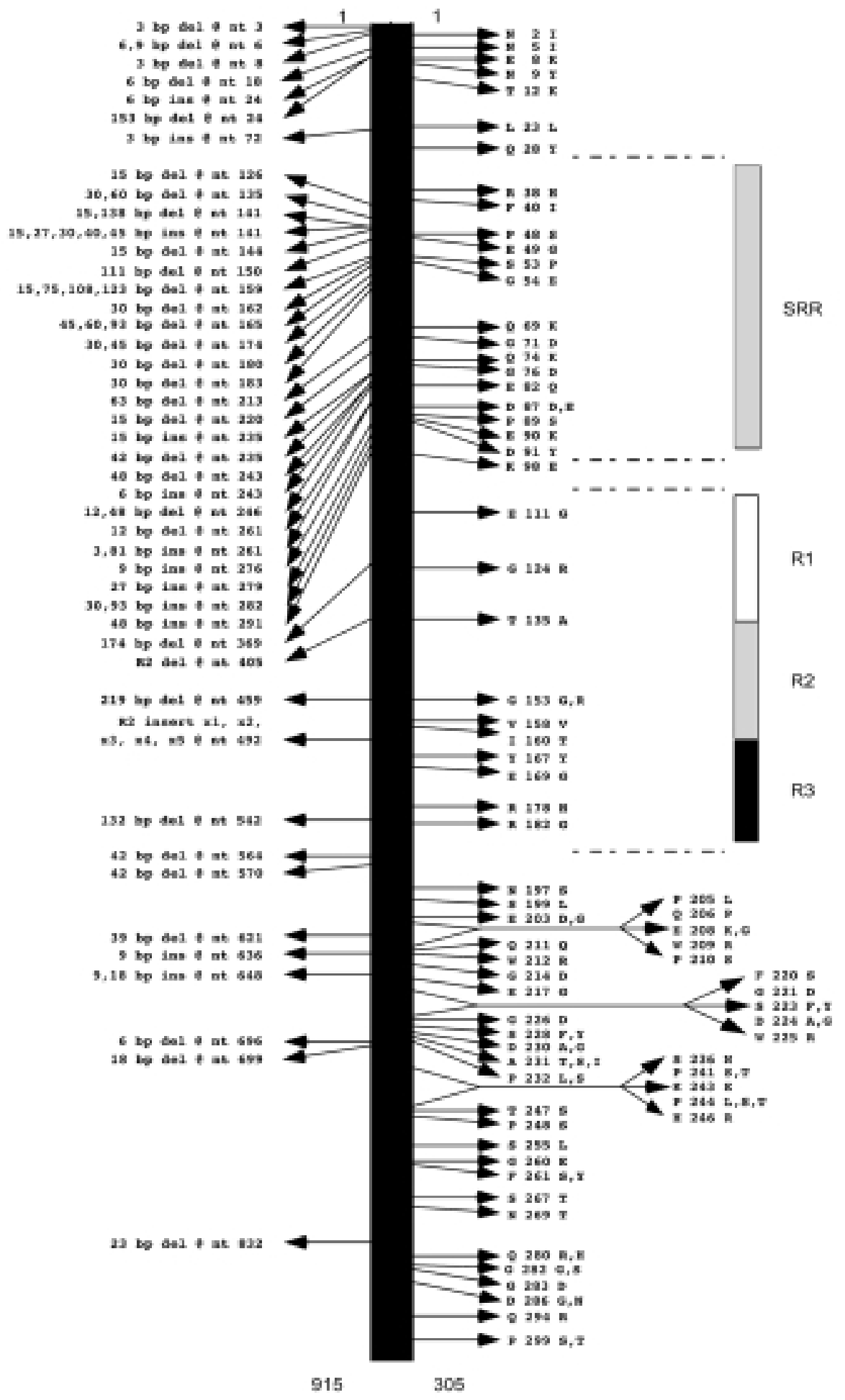

Figure 2

Summary of variation identified in the Sic protein among 1,673 serotype M 1 GAS strains. Left: Positions of the polymorphic nucleotides and amino acids. The single-letter amino acid abbreviations are used. Far right: Domains of Sic located in the proximal half of the protein. SRR, short repeat region; R1-R3, repeats $R 1, R 2$, and $R 3$. M odified from ref. 11 with permission. 


\begin{tabular}{c}
\hline Bacterial pol ymor phisms \\
\hline Martin J . Blaser and James M . M usser, \\
Series Editors
\end{tabular}

Single amino acid polymorphisms and GAS-human interactions

Allelic variation is common among all eukaryotic and prokaryotic organisms, as well as viruses, and is a wellrecognized source of individual differences in genetic composition and behavior. Allelic variation in human genes that results in structural variation in encoded proteins can havea profound effect on phenotypeand overall fitness. For example, variation in theCFTR and SS genes is responsible for cystic fibrosis and sickle cell anemia, respectively. In principle, allelic variation in bacterial virulencegenes could have important biomedical consequences for host-pathogen interactions. There has been little investigation of the potential ramifications of naturally occurring singleamino acid polymorphisms for interactions of the host with bacterial pathogens, including GAS.

An Arg-Gly-Asp (RGD) loop embellishment in an extracellular cysteine protease virulence factor. Stockbauer et al. (8) identified one example illustrating how a single naturally occurring amino acid replacement in a GAS virulence factor alters the character of interac- tion with human cells. GAS isolates produce a highly conserved extracellular cysteine protease known as streptococcal pyrogenic exotoxin B (SpeB) that is a critical virulence factor (20-22). The enzyme detrimentally affects many important host molecules, such as fibronectin, vitronectin, and $\mathrm{H}$-kininogen (20). M otivated by the observation that immunization with SpeB confers protective immunity to mice (22), Stockbauer et al. assessed the level of allelic variation in the speB gene by sequence analysis of the gene from 200 GAS isolates (8). Three main mature $\mathrm{SpeB}$ variants were identified that differed from one another by only one or two amino acids. O ne of these variants (arbitrarily designated $\mathrm{mSpeB} 2$ ) contains an Arg-Gly-Asp (RGD) sequence, a tripeptidemotif that is recognized by a variety of host integrins (Figure 1). Importantly, mSpeB2 is made by all isolates of the unusually abundant serotype M 1 and several other geographically widespread clones that frequently cause invasive infections. Only the $\mathrm{mSpeB2}$ variant bound specifically via the RGD motif region to host cells expressing integrin $\alpha_{v} \beta_{3}$ (also known as the vit-
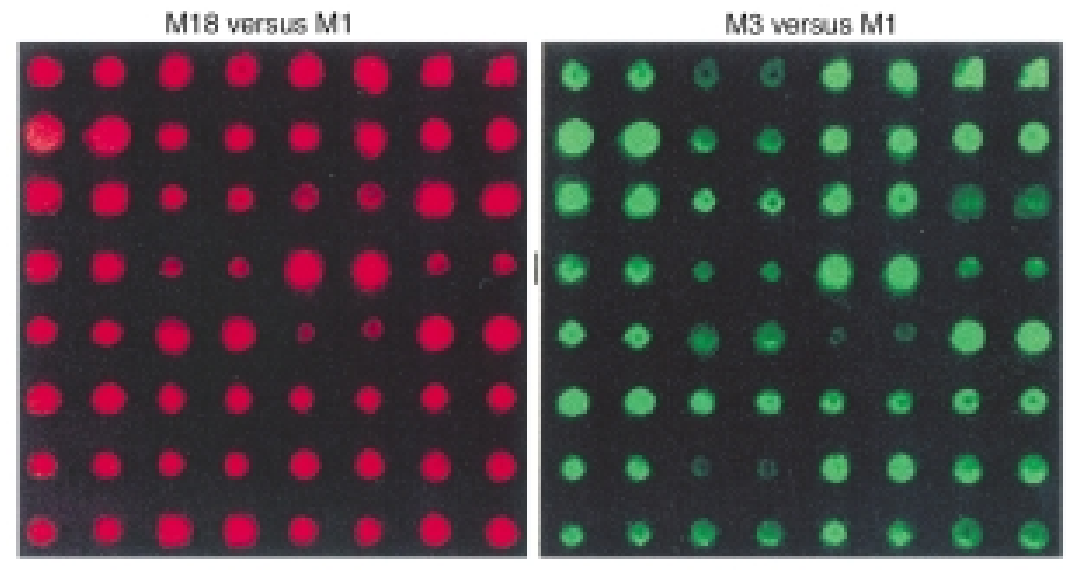

\section{Figure 3}

Comparative genome analysis of three GAS strains with a DNA microarray. The array was constructed with PCR products formulated on the basis of a genome sequence available for a serotype M 1 strain (38). Approximately $99 \%$ of the M 1 open reading frames were represented in duplicate on the array. Shown for illustrative purposes are the hybridization results obtained from only one sector of the entire genome array. Sheared genomic DNA from an M 18 strain and an M 3 strain were labeled with fluorescent dyes $\mathrm{Cy} 5$ and $\mathrm{Cy} 3$, respectively, and hybridized to the $\mathrm{M} 1$ microarray. The hybridization results were analyzed with a ScanArray 5000 laser scanner and QuantArray software package (Packard Biosciences, M eriden, Connecticut, USA). The composite image (bottom) shows that a genomic segment encoding a hypothetical cytosolic protein (green) is unique to this M 3 strain, whereas a genomic segment encoding a hypothetical membrane protein (red) is unique to this M 18 strain. The yellow spots represent DNA that is present in these $M 1, M 3$, and $M 18$ strains.
Composite

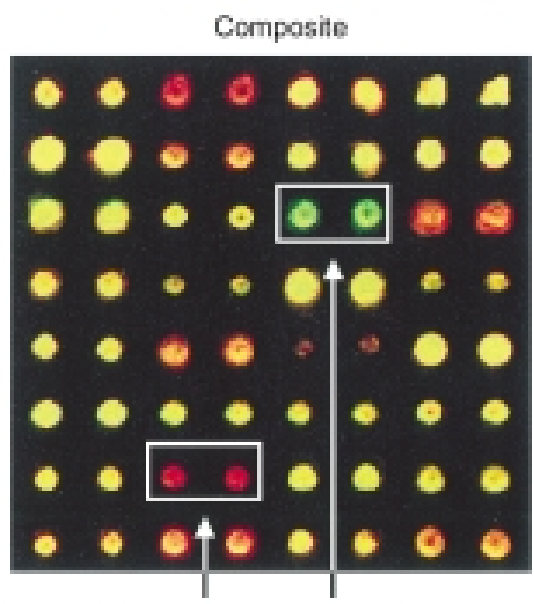

Hypothetical membrane protein gene
Hypothetical cylosolic protein gene 
Table 1

Allelic polymorphism in GAS genes

\begin{tabular}{|c|c|c|c|c|c|}
\hline Protein & Gene & $\begin{array}{l}\text { Function, } \\
\text { proven or putative }\end{array}$ & $\begin{array}{l}\text { No. of } \\
\text { alleles }\end{array}$ & $\begin{array}{l}\text { Biomedical relevance, } \\
\text { proven or putative }\end{array}$ & Reference \\
\hline M protein & emm & Antiphagocytosis & 150 & Serologic classification scheme & (12) \\
\hline M protein type 1 & emm1 & Antiphagocytosis & 37 & Abundance in human infection & (10) \\
\hline M protein type 3 & emm3 & Antiphagocytosis & 32 & Abundance in human infection & $\begin{array}{l}\text { J. Musser, } \\
\text { unpublished data }\end{array}$ \\
\hline Streptococcal pyrogenic exotoxin A & speA & Pyrogenicity, superantigenicity & 6 & $\begin{array}{l}\text { Dissemination and virulence } \\
\text { of allelic variants }\end{array}$ & (13) \\
\hline Streptococcal pyrogenic exotoxin B & speB & Cysteine protease & 89 & $\begin{array}{l}\text { Effects of variation on host- } \\
\text { pathogen interaction }\end{array}$ & (8) \\
\hline Streptococcal pyrogenic exotoxin C & speC & Pyrogenicity, superantigenicity & 4 & Association with invasive disease & (16) \\
\hline Streptococcal inhibitor of complement & sic & Assist persistence in respiratory tract & 296 & Epidemic waves & (10) \\
\hline Streptococcal collagen-like protein & scl & Adherence, soft tissue pathogenesis & 18 & Delayed clearance by host & (18) \\
\hline Streptokinase & skn & Plasminogen activation & 23 & $\begin{array}{l}\text { Diversifying role of intragenic } \\
\text { recombination }\end{array}$ & (7) \\
\hline Hyaluronidase & hyl & Hyaluronic acid degradation & 9 & GAS tissue dissemination & (14) \\
\hline Penicillin-binding protein & pbpla, 2x & Penicillin binding & 1 & Penicillin interaction & $\begin{array}{l}\text { J. Musser, } \\
\text { unpublished data }\end{array}$ \\
\hline Streptococcal superantigen & ssa & Superantigen & 3 & $\begin{array}{l}\text { Toxic shock, diversifying role } \\
\text { of intragenic recombination }\end{array}$ & (15) \\
\hline
\end{tabular}

AThe number of strains studied for each gene varies.

ronectin receptor) or $\alpha / \mathrm{l} b \beta_{3}$ (platel et glycoprotein IIb-IIIa). In essence, analysis of speB allelic variation resulted in (a) identification of the first Gram-positive virulence factor that directly binds integrins, (b) identification of naturally occurring variants of a documented Gram-positive virulencefactor with biomedically relevant differences in their interactions with human cells, and (c) enhanced appreciation that subtle natural variation in the structure of a microbial virulence factor alters the character of hostpathogen interactions. These results stimulated Kagawa et al. (23) to solve the SpeB crystal structure, which revealed the surface location of this integrinbinding RGD motif (Figure 1).

SpeA and the resurgence of invasive GAS disease. SpeA is one of numerous pyrogenic toxins produced by GAS (24). $M$ any of thesetoxins are superantigens that stimulate proliferation of T-cell subsets bearing specific Tcell receptor (TCR) variable domains, a process that can lead to a cytokine storm, shock, and death (24).

Theidentification of STSS, and the significant association of SpeA production with organisms causing this syndrome, led to an interest in allelic variation in speA. Population genetic analysis predicted the likelihood of new variants of speA in part on the basis of the observation that this gene was distributed among strains representing the breadth of genomic diversity in GAS (3). Comparative sequencing of thespeA genein isolates representing a range of ETs identified four alleles, designated speA 1 through speA 4 (9). SpeA2 and SpeA3 each differed from the presumed ancestral vari- ant (SpeA1) by singleamino acid replacements at positions 80 (Gly $\rightarrow$ Ser) and 76 (Val $\rightarrow$ Ile), respectively. Importantly, all contemporary M 1/ET1 and M 3/ET2 strains had the speA2 and speA3 alleles, respectively, whereas strains of these same ETs recovered before 1950 had the ancestral speA 1 allele $(25,26)$.

The association of two variant speA alleles with two widely disseminated GAS clones that have caused the majority of invasive disease episodes in the last two decades suggests that the mutants differ functionalIy. In this regard, Klineand Collins reported (27) that the new SpeA3 toxin variant was more mitogenic and had tenfold higher affinity for the class II M HC molecule than the old SpeA1 variant. Crystal structure analysis revealed that the two amino acid replacements, Gly80Ser in SpeA2 and Val76llein SpeA3, are located close to a zinc-binding sitein $\mathrm{SpeA} 1$, a region postulated to be involved in class II M HC recognition $(28,29)$. Taken together, the results suggest that clones expressing the mutant toxins might have more severe effects in vivo. Although the exact role of SpeA in GAS infections and STSS remains unresolved, it is clear that allelic variants of speA are molecular markers of very successful clones of this human pathogen.

\section{Structural variation in the streptococcal inhibitor of} complement (Sic) protein and GAS epidemic waves In addition to being the most common serotype recovered from invasive disease episodes, M 1 strains display epidemic wave behavior (1). The molecular mechanisms responsiblefor theabundance of $M 1$ organisms 
in invasive disease and the factors contributing to the emergence and perpetuation of epidemic waves are now being revealed. Conventional wisdom and published data led to the concept that most M 1 epidemic waves aremono- or oligoclonal. Recent analysis of allelic variation in the sic virulence gene has proven otherwise $(6,10,11)$ (Figure 2).

Thesic gene encodes an extracellular protein known as streptococcal inhibitor of complement that inhibits thenormal cytolytic function of thecomplement C5bC9 membrane attack complex in vitro (30). This gene is present in $\mathrm{M} 1$ strains but occurs very rarely in other GAS organisms. Analysis of allelic variation in the sic gene in 1,673 M 1 GAS isolates obtained from comprehensive, population-based surveillance studies of invasive disease discovered a strikingly high level of diversity among organisms that are otherwise very closely related genetically (Figure 2$)(6,10,11)$. Hence, M 1 epidemic waves are composed of an array of subclones characterized by Sic variants that are rapidly selected in human populations. Remarkably, virtually all polymorphisms result in structural variation in the Sic protein, indicating that strong positive (D arwinian) selection acts on sic. Several lines of evidence suggest that Sic variants are selected on the mucosal surface by a mechanism involving host antibody (10). Recently, to gain further understanding of the molecular mechanisms of M 1 epidemic waves, we have sequenced thesic gene in GAS isolates causing pharyngitis and invasive disease in metropolitan Helsinki, Finland, recovered during a 37-month populationbased surveillancestudy (31). Our results indicatethat the majority of invasive organisms are represented in the pool of organisms causing pharyngitis in the same geographic area. M oresignificantly, we discovered that new Sic variants occur among pharyngitis isolates an average of 9.8 months prior to their recovery from invasive episodes. These data unambiguously demonstrate mucosal selection of Sic variants. Thesignificant time lapse that occurs between the appearance of an M 1 GAS clone first in noninvasive and subsequently in invasive disease may have implications for the development of a practical strategy to predict epidemics of invasive GAS disease. The mechanism contributing to the abundance of $\mathrm{M} 1$ in human infections involves a process whereby Sic significantly enhances persistence in the mammalian upper respiratory tract (32). Hence, analysis of allelic variation in thesic genehas provided critical new insight into the molecular population genetics of serotype M 1 GAS epidemic waves $(10,31)$. Surprisingly, analysis of allelic variation in the gene (emm1) encoding M 1 protein ruled out the idea that diversity at this locus contributes to these epidemic waves. Similarly, recent results indicate that structural variation in the M 18 protein does not account for variation in the frequency of occurrence of acuterheumatic fever episodes in the region of Salt Lake City, Utah (J.M. M usser, unpublished data).

Future considerations: geno me-scale investigations Currently, GAS genome sequencing projects are nearing completion at the University of Oklahoma (Norman, O klahoma) (serotype M 1), the Sanger Centre (Cambridge, United Kingdom) (serotype M 5), and the Laboratory of Human Bacterial Pathogenesis, Rocky Mountain Laboratories (multiple strains). The volume of resulting data will be extensive, but will the information move us closer to understanding the complexities of GAS pathogenesis and to formulating new therapeutics? Analysis of the available GAS sequence data (commonly known as data mining) has al ready produced new discoveries $(18,19,33)$, and, undoubtedly, more will rapidly accrue. One of the unexpected insights obtained from population genetic analyses of GAS is that alleles of many genes are reassorting more or less at random over loci, as opposed to the clonal nature of many other bacterial pathogens (34). The resultant new combinations of virulence genes are rapidly expressed, and the overall rate of evolution in the species is accelerated compared with that due to simple nucleotide substitutions (1). Comparison of GAS genomes by D N A microarray technology (35) has al ready begun to reveal sequences that are common or unique to distinct strains (Figure 3). Genome comparisons with DNA microarrays are being conducted on a large scale in our laboratory. Use of global genomic and proteomic analysis methods will provide substrates to generate additional testable hypotheses regarding host-GAS interactions, an especially important issue because longdescribed associations of $M$ types with certain clinical conditions such as rheumatic fever defy adequate molecular explanation. In addition, because most or all GAS strains have one or more bacteriophages integrated into their genomes $(36,37)$, it will be critical to assess the extent to which transduction events have mediated interstrain differences in virulence gene complement. We are certain that investigation of GAS allelic variation, population genetics, and host interactions on a genome scale will provide many new insights pertaining to human disease caused by this pathogen.

\section{Acknowledgments}

J.M. Musser wishes to thank R.K. Selander for encouraging and supporting research into the molecular population genetics of human bacterial pathogens. We thank T. Schwan and J. Portis for review of the manuscript and T. Kindt, R.M . Krause, 
and $A$. Fauci for their critical contributions to the GAS research effort at Rocky M ountain Laboratories. Some of the research on GAS conducted by J.M. Musser and described herein was supported by Public Health Services grant Al-33119. We apologize to colleagues whose important work could not be cited due to space limitations.

1. M usser, J.M ., and Krause, R.M. 1998. The revival of group A streptococcal diseases, with a commentary on staphylococcal toxic shock syndrome. In Emerging Infections. R.M. Krause, editor. Academic Press. New York, N ew York, USA. 185-218.

2. Fischetti, V.A. 2000. Surface proteins on Gram-positive bacteria. In Gram-positive pathogens. V.A. Fischetti, R.P. N ovick, J.J. Ferretti, D.A Portnoy, and J.I. Rood, editors. American Society for M icrobiology Press. Washington, DC, USA. 11-24.

3. M usser, J.M ., et al. 1991. Streptococcus pyogenes causing toxic-shocklike syndrome and other invasive diseases: clonal diversity and pyrogenic exotoxin expression. Proc. N atl. Acad. Sci. USA. 88:2668-2672.

4. M usser, J.M. 1996. M olecular population genetic analysis of emerged bacterial pathogens: selected insights. Emerg. Infect. Dis. 2:1-17.

5. Selander, R.K., and M usser, J.M. 1990. The population genetics of bacterial pathogenesis. In M olecular basis of bacterial pathogenesis. B.H. Iglewski and V.L. Clark, editors. Academic Press Inc. Orlando, Florida, USA. 11-36.

6. Hoe, N., et al. 1999. Rapid molecular genetic subtyping of serotype M 1 group A Streptococcus strains. Emerg. Infect. Dis. 5:254-263.

7. Kapur, V., et al. 1995. M olecular population genetic analysis of the streptokinase gene of Streptococcus pyogenes: mosaic alleles generated by recombination. Mol. Microbiol. 16:509-519.

8. Stockbauer, K.E., et al. 1999. A natural variant of the cysteine protease virulence factor of group A Streptococcus with an arginine glycine-aspartic acid (RGD) motif preferentially binds human integrins $\alpha_{\mathrm{v}} \beta 3$ and $\alpha / \mathrm{lb} \beta 3$. Proc. Natl. A cad. Sci. USA. 96:242-247.

9. N el son, K., Schlievert, P.M., Selander, R.K., and M usser, J.M. 1991. Characterization and clonal distribution of four alleles of the speA gene encoding pyrogenic exotoxin A (scarlet fever toxin) in Streptococcus pyogenes. J. Exp. M ed. 174:1271-1274.

10. Hoe, N .P., et al. 1999. Rapid selection of complement-inhibiting protein variants in group A Streptococcus epidemic waves. Nat. M ed. 5:924-929.

11. Stockbauer, K.E., et al. 1998. H ypervariability generated by natural selection in an extracellular complement-inhibiting protein of serotype M 1 strains of group A Streptococcus. Proc. N atl. A cad. Sci. USA 95:3128-3133.

12. Facklam, R., et al. 1999. emm typing and validation of provisional $M$ types for group A streptococci. Emerg. Infect. Dis. 5:247-253.

13. Bessen, D.E., et al. 1999. Genetic linkage of exotoxin alleles and emm gene markers for tissue tropism in group A streptococci. J. Infect. Dis. 179:627-636.

14. M arciel, A.M., Kapur, V., and M usser, J.M. 1997. M olecular population genetic analysis of a Streptococcus pyogenes bacteriophage-encod ed hyaluronidase gene: recombination contributes to allelic variation. M icrob. Pathog. 22:209-217.

15. Reda, K.B., et al. 1996. Phylogenetic distribution of streptococcal superantigen SSA allelic variants provides evidence for horizontal transfer of ssa within Streptococcus pyogenes. Infect. Immun. 64:1161-1165.

16. N orrby-Teglund, A., Holm, S.E., and N orgren, M . 1994. Detection and nucleotide sequence analysis of the speC gene in Swedish clinical group A streptococcal isolates. J. Clin. Microbiol. 32:705-709.

17. Kapur, V., N el son, K., Schlievert, P.M., Selander, R.K., and M usser, J.M. 1992. Molecular population genetic evidence of horizontal spread of two alleles of the pyrogenic exotoxin $C$ gene (speC) among pathogenic clones of Streptococcus pyogenes. Infect. Immun.
60:3513-3517.

18. Lukomski, S., et al. 2000. Identification and characterization of the scl gene encoding a group A Streptococcus extracellular protein virulence factor with similarity to human collagen. Infect. Immun. 68:6542-6553.

19. Lukomski, S et al. 2001. I dentification and characterization of a second extracellular collagen-like protein made by Group A Streptococcus: control of production at the level of translation. Infect. Immun. In press.

20. M usser, J.M . 1997. Streptococcal superantigen, mitogenic factor, and pyrogenic exotoxin B expressed by Streptococcus pyogenes. Structure and function. Prep. Biochem. Biotechnol. 27:143-172.

21. Lukomski, S., et al. 1997. Inactivation of Streptococcus pyogenes extracellular cysteine protease significantly decreases mouse lethality of serotype M 3 and M 49 strains. J. Clin. Invest. 99:2574-2580.

22. Kapur, V., et al. 1994. Vaccination with streptococcal extracellular cysteine protease (interleukin-1 beta convertase) protects mice against challenge with heterologous group A streptococci. Microb. Pathog. 16:443-450.

23. Kagawa, T.F., et al. 2000. Crystal structure of the zymogen form of the group A Streptococcus virulence factor SpeB: an integrin-binding cysteine protease. Proc. N atl. A cad. Sci. USA. 97:2235-2240.

24. Schlievert, P.M. 1993. Role of superantigens in human disease. J. Infect. Dis. 167:997-1002.

25. M usser, J.M ., et al. 1993. Geographic and temporal distribution and molecular characterization of two highly pathogenic clones of Streptococcus pyogenes expressing allelic variants of pyrogenic exotoxin A (scarlet fever toxin). J. Infect. Dis. 167:337-346.

26. M usser, J.M., et al. 1995. Genetic diversity and relationships among Streptococcus pyogenes strains expressing serotype M 1 protein: recent intercontinental spread of a subclone causing episodes of invasive disease. Infect. Immun. 63:994-1003.

27. Kline, J.B., and Collins, C.M. 1996. Analysis of the superantigenic activity of mutant and allelic forms of streptococcal pyrogenic exotoxin A. Infect. Immun. 64:861-869.

28. Papageorgiou, A.C., et al. 1999. Structural basis for the recognition of superantigen streptococcal pyrogenic exotoxin A (SpeA1) by M HC class II molecules and T-cell receptors. EM BO J. 18:9-21.

29. Earhart C., Vath G., Roggiani M., Schlievert P., and Ohlendorf D. 2000. Structure of streptococcal pyrogenic exotoxin A reveals a novel metal cluster. Protein Sci. 9:1847-1851.

30. Åkesson, P., Sjöholm, A.G., and Björck, L. 1996. Protein SIC, a novel extracellular protein of Streptococcus pyogenes interfering with complement function. J. Biol. Chem. 271:1081-1088.

31. Hoe, N.P., et al. 2001. Distribution of streptococcal inhibitor of complement variants in pharyngitis and invasive isolates in an epidemic of serotype M 1 group A Streptococcus infection. J. Infect. Dis. 183:633-639.

32. Lukomski, S., et al. 2000. N onpolar inactivation of the hypervariable streptococcal inhibitor of complement gene (sic) in serotype M 1 Streptococcus pyogenes significantly decreases mouse mucosal colonization. Infect. I mmun. 68:535-542.

33. Rasmussen, M., M üller, H.-P., and Björck, L. 1999. Protein GRAB of Streptococcus pyogenes regulates proteolysis at the bacterial cell surface by binding $\alpha_{2}$-macroglobulin. J. Biol. Chem. 274:15336-15344.

34. Kehoe, M.A., Kapur, V., Whatmore, A.M ., and M usser, J.M. 1996. Horizontal gene transfer among group A streptococci: implications for pathogenesis and epidemiology. Trends M icrobiol. 4:436-443.

35. Schena, M., Shalon, D., Davis, R.W., and Brown, P.O 1995. Quantitative monitoring of gene expression patterns with a complementary D N A microarray. Science. 270:467-470.

36. O hlsen, K., et al. 1999. M obile elements, phages, and genomic islands of staphylococci and streptococci. In Pathogenicity islands and other mobile virulence elements. J.B. Kaper and J. H acker, editors. American Society for M icrobiology Press. Washington, DC, USA. 265-287.

37. Krause, R.M. 1957. Studies on bacteriophages of hemolytic streptococci. J. Exp. Med. 106:365-384.

38.Streptococcus pyogenes Genome Sequencing Project. http://www.genome.ou.edu/strep.html. 